\title{
PEYRONIE'S DISEASE: CURRENT STATUS AND NEW DEVELOPMENT
}

\author{
MD. ABUL HOSSAIN ${ }^{1}$, MD. WALIUL ISLAM ${ }^{2}$, MD. NURUL HOODA ${ }^{2}$, MD. FAZAL NASER ${ }^{1}$, MD. SHOHRAB \\ HOSSAIN $^{1}$, MD. SHAFIQUL AZAM ${ }^{1}$ \\ ${ }^{1}$ Department of Urology, Shaheed Suhrawardy Medical College \& Hospital, ${ }^{2}$ National Institute of Kidney Diseases \& Urology
}

\begin{abstract}
:
Objective: To assess the articles published on current treatments for Peyronie's disease $(P D)$ and it's new development and to assist clinicians to select the effective management of PD by increasing understanding and awareness of the outcomes associated with current medical and surgical treatment options.
\end{abstract}

Methods: A Hinari literature search was conducted to identify relevant, peer-reviewed, clinical and review articles published related to current treatment options and it's new developments of Peyronie's Disease. Search terms for this non-systematic review included 'Peyronie's disease', 'current treatment, outcomes', new development. 'Erectile dysfunction or ED', search terms were searched separately and in combination. Case studies and editorials were excluded, primary manuscripts and reviews were included, and references of articles of interest were reviewed and key references were obtained.

Result: Currently, there are several investigational minimally invasive and non-surgical treatment options for PD; however, surgical treatment remains the standard of care for patients with stable disease and disabling deformity or drug-resistant erectile dysfunction. Each of the different surgical procedures that are used for treatment of PD, including tunical shortening, tunical lengthening (plaque incisions or partial excision and grafting), and use of inflatable penile prostheses, carries its own advantages and disadvantages in terms of potential complications and postoperative satisfaction. No single, standard, surgical treatment for this disorder has prevailed and multiple variations of each type of procedure exist. Obtaining data on current treatment and its modifications to these procedures, and new surgical techniques and materials may serve to further guide

Conclusion: The real etiology of Peyronie's disease and the mechanisms of formation of the plaque still remain obscure. Although conservative management is obtaining a progressively larger consensus among the experts, surgical correction still remains the mainstay treatment for this condition. Treatment should be tailored to each patient after a detailed evaluation of disease severity and sexual function.

Keywords: Peyronie's disease, Medical treatment, Surgical treatment, New development, erectile dysfunction.

Bangladesh J. Urol. 2014; 17(2): 87-96

\section{Introduction:}

The name "Peyronie's Disease" is derived from the physician Francois Gigot de la Peyronie, personal physician to King Louis XV of France. De la Peyronie wrote an authoritative description of the disorder in 1743 and his name has been associated with the condition

Correspondence : Md. Abul Hossain, Department of Urology, Shaheed Suhrawardy Medical College, Email: drmahossain.qpm@gmail.com since that time. Peyronie's disease (PD) is the development of fibrous tissue inside the penis that causes painful erection .Peyronie's disease (PD) is a progressive, two-phase medical condition where disordered collagen deposition replaces the normal elastic fibres of the tunica albuginea, resulting in palpable collagen plaque development in the tunica albuginea of the penis[1]. The impact of peyronie's disease is 
extensive in terms of personal self-esteem and sexual function. Only in the past few years have new concepts emerged that should spur a greater basic research effort and ultimately, improvement in management of this condition.

\section{Etiology:}

$\mathrm{PD}$ is a benign, acquired connective tissue disorder. The exact etiology of PD still remains vague despite its description many centuries ago. There seems to be interplay between genetic predisposition, autoimmunity, trauma and inflammation[2]. Injury, recurrent microtrauma and a disorder in wound healing are major etiologies for the developmentof a penile plaque [3].

\section{Incidence:}

Multiple demographic studies have been performed worldwide indicating a preva-lence rate of 3-9\% in adult men. Therefore PD is not a rare disorder. Recent studies suggest a prevalence of PD in the population that can reach up to $9 \%[4,5]$, much higher than initially thought. The mean age of onset of the condition in these studies was 55-60 years, and penile curvature was present in over $80 \%$ of patients whereas painful erection was reported by over half of them[6-8].

The estimated prevalence rates for PD among men in the general population range from 3 to 9\% [9-11]. Traditionally, PD has been considered a rare disorder; however, it is possible that its prevalence has been underreported as men may not seek treatment because of embarrassment or a lack of awareness of available treatments, or because the symptoms are not disabling enough in the patient's opinion to warrant treatment. The incidence of PD was reported as $3.2 \%$ in the Cologne male survey consisting of 8,000 men. Other epidemiological studies estimate the prevalence of $\mathrm{PD}$ to be 3-8.9\% with mean age of onset being 50-60 years, [12] in addition, recent studies have reported up to $10.8 \%$ prevalence of PD in men younger than 40 years who display a more acute onset and lower incidence of ED compared to older men $[13,15]$.

\section{Natural history:}

The natural history of PD has been evaluated in only a few level 2 and 3 studies indicating that spontaneous deformity resolution is not common. The course of the disease can be variable, with up to $13 \%$ regressing spontaneously, $40 \%$ progressing if untreated, and $47 \%$ showing no change over time [16].

\section{Pathology:}

Actual pathophysiology of PD are poorly delineated. The pathological outcome is a fibrous, inelastic tunical plaque(s) with excess abnormal collagen and lossof elastin. The main pathological process is tissue fibrosis with disorganization of elastic fibers, combined in most cases with fibrin accumulation and different degrees of inflammation [17]. Myofibroblasts were first identified in, and cultured from, PD plaques in 1988. Despite the assumption that myofibroblasts have an important role in PD and abundant evidence for their involvement in Dupuytren's disease, these findings were not followed up for another two decades. Only in the past 4 years have the following observations been made: first, that myofibroblasts are present in normal human tunica albuginea, but exist in considerably greater numbers in PD plaques (detected as relative expression of vimentin, a marker of fibroblasts [which differentiate to form myofibroblasts], and $\alpha$-smooth-muscle actin, a marker of myofibroblasts), [18-19]; second, that this differential expression is mimicked in TGF- $\alpha 1$-induced and fibrininduced rat models of PD [20-21] and third, that fibroblast cultures derived from unaffected human tunica and PD plaques differentiate into myofibroblasts, synthesize collagen and undergo apoptosis. Their gene-expression profiles reflect the processes that contribute to in vivo development of PD in men and rat models [22]. Peyronie's disease plaques result from dynamic interplay between profibrotic and antifibrotic factors in cells such as fibroblasts and myofibroblasts of the tunica albuginea. One of the antifibrotic mechanisms in the Peyronie's disease plaque is increased levels of nitric oxide and cyclic GMP resulting from sustained expression of inducible nitric oxide synthase. Corporal fibrosis, like tunical fibrosis, seems to be counteracted by inducible nitric oxide synthase.

Recent studies on an animal model suggest that transforming growth factor beta (TGF b1)[23] and myofibroblasts [24-27] play an important role in the formation of PD plaques. TGFb1 is also found in the human PD plaque and is the main profibrotic factor in multiple tissues [28], while myofibro-blasts are a common feature in most tissue fibrosis and in abnormal wound healing [29], and their persistence by the inhibition of programmed cell death leads to scar formation [30]. Moreover, the tunica albuginea is known to contain pluripotent stem cells that are potentially able to differentiate into myofibroblasts, smooth muscle cells, and osteoblasts, and in a paracrine fashion to modulate the differentiation of a multipotent cell line into 
osteoblasts and myofibroblasts [31-34]. The presence of stem cells in the normal tunica albug-inea may explain the fibrotic and osteogenic pro-gression of the PD plaque upon the release of cytokines following microtrauma to the penis that would stimulate this cell lineage commitment

\section{Phases of peyronie's disease:}

Generally, PD is thought to have an acute inflammatory phase followed by a chronic phase. The acute (active) phase of PD ischaracterized by penile inflammation, occasionally associatedwith pain on palpation with erections and/or during sexualactivity, and progression in plaque size and curvature deformity [35]. In most cases, the duration of this phase is 12 months from onset of the disease. PD progressively deteriorates if left untreated in the acute phase. Resolution of inflammation and stabilizationof plaque size and curvature deformity mark the chronic (stable) phase of disease [36]. Accordingly, Mulhall et al.[37] reported the results of 246 patients who presented within 6 months of disease onset and were followed-up for one year without treatment. The authors demonstrated that penile curvature worsened in almost half of the cohort, while only $12 \%$ of men demonstrated improvement in terms of mean degree of curvature during the follow-up period. The chronic phase, during which penile curvature and plaque size remain stable in the absence of penile pain, is generally accepted to commence 12 months from onset [38].

\section{Clinical presentation:}

A cute phase symptoms:

Patients with short disease duration ( $<12$ months), penile pain, or a recent change in penile deformity are still likely to have active inflammatory disease. Penile pain may be persistent in the inflammatory stage of the disease but is usually only present during erection. The pain is not usually severe in nature but may interfere with sexual function [39- 40]. ED associated with PD has been reported in up to $58 \%$ of men [41].

\section{The chronic phase symptoms:}

During which penile curvature and plaque size remain stable in the absence of penile pain, is generally accepted to commence 12 months from onset. The diagnosis of PD should be based on a comprehensive medical and sexual history and a detailed physical examination [42].

\section{Physical examination:}

Men with PD can have either a well-defined plaque or a palpable indurations. Two-thirds of patients have a palpable induration on the dorsal aspect of the penis with corresponding penile curvature. ventrally-located and laterally- located plaques are less common, with an incidence of $10-20 \%$ and 6-10\%, respectively. Measurement of stretched penile length is an important feature of the physical examination.[43]

\section{Assessment of lesion severity}

Erectile capacity is best assessed through a detailed patient history that includes the completion of a validated erectile function questionnaire (International Index of Erectile Function (IIEF ) or a cavernosal injection and self- stimulation (CIS) test may be performed using vasoactive agents. In addition to assessing erectile capacity, the CIS test may provide the most accurate means of measuring penile curvature[44].

\section{Common comorbidities:}

Multiple comorbidities have been identified, including ED, hypertension, diabetes mellitus, hyperlipidenia, low testosterone, and Dupuytren's disease. It remains unclear whether any of these contribute to the development of PD.

Ageing, hypertension and diabetes are associated with $P D$, as well as with ED, which affects $20-54 \%$ of men with PD; [45]; however, no correlation has been found between the severity of penile curvature and any of these comorbidities. About $15-20 \%$ of PD patients present with dupuytren's disease, which is characterized by fibrotic nodules in the palmar fascia [46].

\section{Investigations in PD:}

Ultrasound is used to identify the site and consistency of the plaque and is a useful tool to determine the extent of plaque. A vascular assessment should be performed in all patients with ED as well as those undergoing surgery and is best done using duplex ultrasound [47]. It is a useful but not a necessary test.

\section{Treatment of peyronie's disease:}

Different treatment options exist for PD and numerous methods have been described with varying levels of success, including oral medication, intralesional injection, topical agents, and even extracorporeal shockwave therapy as well as surgery [48]. Treatments should be tailored for each patient according to the condition of the disease with the ultimate goal being a satisfactory erection for the patient. 


\section{Medical therapy:}

A wide range of medical therapies have been utilized for the treatment of Peyronie's disease. Because there is the lack of a clear understanding of the etiopathophysiology, so a cure has not been found. Therefore, a variety of treatment options have been used.

\section{Candidates for medical therapy:}

Men with early phase disease (i.e., <12 months induration) manifest by unstable or progressive deformity and painful erections as well as those not psychologically ready or interested in surgery may be considered candidates for nonsurgical therapy.

\section{Vitamin E :}

Vitamin $E$ is the most common nonsurgical therapy prescribed by urologists for treating PD [49]. Vitamin E is thought to be active through its antioxidant properties as has been demonstrated in numerous in vitro studies. It is unclear, however, whether these effects are significant in human studies[50].

\section{Tamoxifen:}

Tamoxifen is a nonsteroidal antiestrogen whose mechanism of action with regard to PD treatment remains unclear. It may act through a reduction in the production of TGF-b by fibroblasts in the tunica albuginea. Administration of Tamoxifen $20 \mathrm{mg}$ twice daily can induce significant improvement in penile pain, curvature, and plaque size in the early stages of the disease [51]. However, these encouraging results have not been confirmed by recent studies [52].

\section{Colchicine:}

Although initial studies showed that colchicines might be also effective in the early phase of the disease according to the finding of two recent studies $[53,54]$ recent series have showed that colchicine is no better than placebo [55].

\section{Pentoxifylline:}

Pentoxifylline is a nonspecific PDE-5 inhibitor used for a wide range of medical applications. Pentoxifylline added to fibroblast culture resulted in an upregulation of CAMP and decreased collagen I production, suggesting potential benefit for the abnormal collagen production seen in PD. Additional in vitro evidence revealed anion of osteoclastic activity after treatment with pentoxifylline, but the significance of this finding in the corpora cavernosa is unclear [56]. Increased levels of nitric oxide levels may be effective in preventing progression of PD or reversing its fibrosis as described by Brant et al. [57].

\section{Potaba:}

Potaba (potassium paraaminobenzoate) likely exerts its effects through antifibrotic and anti-inflammatory mechanisms. It has been shown in observational studies to be an effective treatment for PD $[58,59]$. Potaba treatment was associated with a $74 \%$ decrease in plaque

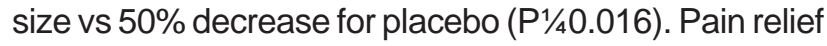
was experienced equally in both groups and no difference in adverse effects was noted.

\section{L-carnitine:}

L-carnitine is hypothesized to act by increasing mitochondrial respiration and decreasing free radical formation[60]. Reported side effects have included mild euphoria and gastrointestinal upset with larger doses (44 g per day). The results of randomized trials have been mixed. One trial comparing tamoxifen vs L-carnitine demonstrated decreased plaque size and curvature in the L-carnitine arm and found that L-carnitine provided great pain reduction and fewer side effects than tamoxifen [61].

\section{Intralesional Injections: \\ Steroids:}

Although some studies using injectable corticosteroids for the treatment of PD showed positive outcomes from treatment, the authors believe that the therapeutic effects were because of the mechanical effects of the injection and not to the drug action itself [62].

\section{Collagenase:}

Although the prospective, randomized, placebocontrolled study of Gelbard failed to demonstrate any clinical benefit with the use of intralesional injections of collagenase, a recent study has reported significant decreases in deviation angle, in plaque width and length [63].

\section{Verapamil:}

In vitro studies verapamil has been shown to interfere with Peyronie's plaque derived fibroblast cellular proliferation and Levine et al. reported that intralesional verapamil injection induces a significant reduction in penile curvature [64]. These encouraging results have been confirmed by two subsequent studies, while one failed to demonstrate any effectiveness of this treatment intralesional Verapamil injections could be recommended for the treatment of noncalcified acute or chronic plaques to stabilize disease progression or possibly reduce penile deformity. 


\section{Interferon:}

A placebo-controlled parallel study showed that intralesional interferon alpha-2 $\beta$ may be beneficial for men with PD [65]. However, a recent study failed to demonstrate any efficacy of intralesional injection.

\section{Surgery:}

Failure in improvement of erections or insufficient erections for intercourse with medical methods is an indication for surgical treatment in men who desire surgery and acceptable candidates. The timing of surgery is important; the course of PD in most patients includes an active and a quiescent phase. Surgery should be avoided in the active phase while the penile deformity develops. Although urologists commonly wait 6 months from the onset of the quiescent phase of PD before performing any procedure, it is best to wait at least 1 year based on the experience of Montorsi et al [66].

Table-I

Indications for surgery

- Stable disease (6 months with no pain and stable deformity)

- Compromised or inability to engage in coitus

- Extensive plaque calcification

- Failed conservative treatment

- Wants the most rapid and reliable result

\section{Surgical options:}

There are three general categories of surgical options for PD; tunical shortening, tunical lengthening/graft, and penile prostheses [67].

\section{Decision making to choice an appropriate option:}

Some form of tunica plication procedure is best for those with curvature less than $60^{\circ}$ and with no hour-glass deformity resulting in a hinge effect. For those with more severe deformity ( $>60^{\circ}$ and/or hourglass) and good preoperative rigidity, incision or partial excision and grafting is recommended.

Penile prosthesis implantation with additional maneuvers to correct the deformity is recommended when there is preoperative ED not responsive to oral medication (phosphodiesterase type 5 Inhibitors)

\section{Tunical shortening procedure:}

\section{The Nesbit Procedure:}

This was first described by Nesbit for treating erectile deformities caused by congenital abnormalities [68] and later applied to PD by Pryor and Fitzpatrick [69]. This procedure has a high success rate, with $82 \%$ of patients in a large retrospective study able to have intercourse with minimal residual penile deformity; $88 \%$ of patients were satisfied with the outcome of the Nesbit procedure in a separate large study, with $82 \%$ having a completely straight penis afterward [70].

The procedure involves excision and plication of the tunica opposite the Peyronie's plaque, thus straightening the penis. A circumcision incision is made and the penile skin degloved. An artificial erection is induced by intracorporal saline injection. The area of maximum bend is marked (pen or suture) on the convex side of the penis (opposite the plaque), and a 5-10 $\mathrm{mm}$ transverse ellipse of the tunica albuginea excised. The ellipse excised should be $1 \mathrm{~mm}$ wide for every $10^{\circ}$ of curvature the penis.

This is day-surgery and patients may be discharged after voiding, and areadvised to avoid intercourse for 6 weeks while they heal. Although the results with the Nesbit procedure are excellent for penis straightening, penile shortening occurs in most patients and is a drawback for many men. Shortening rarely prevents sexual intercourse (1.3-11.9\%) [71]. Complications with the Nesbit procedure include recurrent curvature (7.7$10.6 \%$ of $\left.>30^{\circ}\right)$, ED (3.25-22.9\%), penile haematoma (0$8.9 \%)$, penile narrowing or induration (0-16.7\%), urethral injury (0-1.4\%), suture granuloma (0-1.9\%), penile or glans hypoesthesia (0-21.4\%) and phimosis (0-4.8\%) [72]. This procedure is still a very good option for men with adequate penile length, good erectile function, curvature of more than $45^{\circ}$, and no hour-glass deformity.

\section{The Modified Nesbit Procedure:}

Yachia [73] proposed modifications to the Nesbit procedure to decrease the likelihood of disrupting the neurovascular bundle and subsequent glans hypoesthesia. He proposed making a single longitudinal $(1-1.5 \mathrm{~cm})$ incision or several smaller longitudinal incisions on the convex side of the tunica, following a similar approach as described above. The incisions are then closed horizontally with buried knots using either absorbable or permanent suture, applying the HeinekeMikulicz principle. The procedure has a high rate of satisfaction in various reports [74], although penile shortening remains a common drawback (57-67\%), the highest rate of satisfaction (83\%) and lowest ED (0\%) were with the modified Nesbit. Complications may be similar to those of the traditional Nesbit procedure and glans hypoesthesia is still possible. erections, $32 \%$ with glans hypoesthesia. 


\section{Tunical Lengthening Procedures:}

Incision or excision of Peyronie's plaques and placing grafts has been used for patients with severe penile curvature when other operations could result in a shortened, deformed penis, or when patients have narrowing or hour-glass deformities. The search for the ideal graft continues. As of this time, no ideal graft has been identified, which would take reliably, not contract, be resistant to infection and preserve erectile capacity [75].

Currently, it appears that the nature of the graft is less likely the determining factor with respect to postoperative ED.

Table-II

Graft types

I. Autologous

- Dermis

- Vein

- Temporalis fascia

- Tunica vaginalis

- Buccal mucosa

II. Nonautologous; allografts

- Processed human cadaveric

- Pericardium

- Dermis

- Fascia lata

- Dura mater

III - Processed animal grafts; xenografts

- Bovine pericardium

- Porcine small intestinal submucosa

- Porcine dermis

The most frequently used autologous graft currently in use is saphenous vein, which requires a separate incision to harvest, adding a risk of local side effects, and longer operating time.

The operative procedure is done essentially the same for all grafting techniques - an artificial erection is created demonstrating the curvature and the penis is typically degloved using a circumcising incision allowing exposure of the entire shaft of the penis. In the area of maximum curvature, Buck's fascia containing the neurovascular bundle is elevated, either from a pair of parallel incisions lateral to the urethral ridge allowing elevation of Buck's fascia dorsally, or by coming through the bed of the deep dorsal vein. An artificial erection is recreated, demonstrating the area of maximum deformity. Surgeons differ in their approach as to whether a simple modified $\mathrm{H}$-like incision should be made to the area of maximum curvature or whether partial plaque excision is recommended. Once the graft is positioned, Buck's fascia is reapproximated to provide support and a vascularized cover over the graft.

Reported complications include haematoma (0-4.5\%), penile or glans hypoesthesia (0-16.7\%), shortening (0$40 \%)$, curve recurrence (0-16.7\%) and ED (0-66.7\%) $[76,77]$. Penile or glans hypoesthesia, related to injury to the dorsal penile nerves, frequently resolves after 6 12 months, although it may be lasting. Avoiding penile shortening, associated with the Nesbit and plication procedures, is a common reason for choosing graft procedures.

\section{Penile prosthesis:}

Penile prostheses are the standard of care in patients with ED and penile curvature or severe penile defects that require grafting to repair with or without preexisting $\mathrm{ED}$ [78]. After placing the prosthesis, if residual curvature is more than $45^{\circ}$ additional maneuvers are necessary to straighten the penis. Incision of plaque followed by graft is one effective approach. [79]. Implanting a penile prosthesis, preferably an inflatable type, is a good option in men who have both PD and ED. Montorsi et al. [80] reported poor long-term patient satisfaction with implantation of semirigid prostheses for ED and PD. The average lifespan of a prosthesis is approximately 10 years,with a $4 \%$ mechanical failure at 5 years and $40 \%$ failure by 15 years. Success as defined by complete penile straightening ranges from 80 to $93 \%[81]$.

\section{Conclusion:}

Potaba and L-carnitine treatment are the best supported oral medications, but evidence is far from convincing. Effective treatment with intralesional verapamil has been supported by the largest number of RCTs. New effective therapies are needed that are easy to administer, low cost and have few side effects.

The surgical treatment of Peyronie's disease is a viable and recommended alternative for men with compromised sexual function due to severe curvature or a lesion causing penile instability. The choice of corrective procedure should be tailored to each patient after completing a detailed evaluation of disease severity and sexual function.

Conflict of interest: None declared. 


\section{References:}

1. Ralph D,Gonzalez-Cadavid N,Mirone V et al. The management of Peyronie's disease: evlidence based guidelines. J Sex Med 2010;7:2359-74.

2. Gonzalez-Cadavid NF, Rajfer J. Mechanisms of disease: new insights intothe cellular and molecular pathology of Peyronie's disease. Nat Clin Pract Urol 2005; 2: 291-7.

3. Serap Gur, Ma Limin \& Wayne JG Hellstrom; Current status and newdevelopments in Peyronie'sdisease: medical, minimallyinvasive and surgical treatment options. Expert Opin. Pharmacother. (2011) 12(6):931-944.

4. Mulhall, J. P. et al. Subjective and objective analysis of the prevalence of Peyronie's disease in a population of men presenting for prostate cancer screening. J. Urol. 171, 2350-2353 (2004).

5. Levine, L. A., estrada, C. R., Storm, D. w. \& Matkov, T. G. Peyronie disease in younger men: characteristics and treatment results. J. Androl. 24, 27-32 (2003).

6. Mulhall JP, Creech SD, Boorjian SA, Ghaly S, Kim ED, Moty A, Davis R, Hellstrom W. Subjective and objective analysis of the prevalence of Peyronie's disease in a population of men presenting for prostate cancer screening. J Urol 2004;171: 2350-3.

7. El-Sakka Al. Prevalence of Peyronie's disease among patients, with erectile dysfunction. Eur Urol 2006;49:564-69.

8. Perimenis P, Athanasopoulos A, Gyftopoulos K, Katsenis G, Barbalias G. Peyronie's disease: Epidemiology and clinical presentation of 134 cases. Int Urol Nephrol 2001;32:691-4.

9. Bjekic MD, Vlajinac HD, Sipetic SB, Marinkovic JM. Risk factors for Peyronie's disease: A casecontrol study. BJU Int 2006;97:570-4.

10. Ralph, D. et al. The management of Peyronie's, disease: evidence based 2010 guidelines. J. Sex. Med. 7, 2359-2374 (2010).

11. Deveci, S. et al. Defining the clinical, characteristics of Peyronie's disease in young men. J. Sex. Med. 4, 485-490 (2007).

12. Schwarzer, U. et al. The prevalence of Peyronie's disease: results of a large survey. BJU Int. 88, 727-730 (2001).
13. Levine, L. A., estrada, C. R., Storm, D. w. \& Matkov, T. G. Peyronie disease in younger men: characteristics and treatment results. J. Androl.24, 27-32 (2003).

14. Deveci, S. et al. Defining the clinical characteristics of Peyronie's disease in young men. J. Sex. Med. 4, 485-490 (2007).

15. Kadioglu, A. \& Sanli, O. in Peyronie's disease: a guide to clinical management (ed. Levine, L. A.) 9-18 (Humana Press, Totowa, 2007).

16. Gelbard MK, Dorey F, James K. The natural history of Peyronie's disease. J Urol 1990; 144 : 1376-9

17. Vernet D, Ferrini MG, Valente EG, Magee TR, BouGharios G, Rajfer J, Gonzalez-Cadavid NF. Effect of nitric oxide on the differentiation of fibroblasts into myofibroblasts in the Peyronie's fibrotic plaque and in its rat model. Nitric Oxide 2002;7:262-76.

18. Ferrini MG et al. (2002) Antifibrotic role of inducible nitric oxide synthase. Nitric Oxide 6: 283-294

19. Vernet $\mathrm{D}$ et al. (2002) Effect of nitric oxide on the differentiation of fibroblasts into myofibroblasts in the Peyronie's fibrotic plaque and in its rat model. Nitric Oxide 7: 262-276.

20. Davila $\mathrm{HH}$ et al. (2003) Fibrin as an inducer of fibrosis in the tunica albuginea of the rat: a new animal model of Peyronie's disease. BJU Int 91: 830-838.

21. Valente EG et al. (2003) PDE L-arginine and PDE inhibitors counteract fibrosis in the Peyronie's fibrotic plaque and related fibroblast cultures. Nitric Oxide 9: 229-244.

22. Gonzalez-Cadavid NF and Rajfer J (2004) Therapy of erectile function: potential future treatments. Endocrine 23: 167-176.

23. El-Sakka Al, Hassoba HM, Chui RM, Bhatnagar RS, Dahiya R, Lue TF. An animal model of Peyronie's-like condition associated with an increase of transforming growth factor beta mRNA and protein expression. J Urol 1997;158:2284-90.

24. Somers KD, Dawson DM, Wright GL Jr, Leffell MS, Rowe MJ, Bluemink GG, Vande Berg JS, Gleischman SH, Devine CJ Jr, Horton CE. Cell culture of Peyronie's disease plaque and normal penile tissue. J Urol 1982;127:585-8. 
25. Mulhall JP, Nicholson B, Pierpaoli S, Lubrano T, Shankey TV. Chromosomal instability is demonstrated by fibroblasts derived from the tunica of men with Peyronie's disease. Int J Impot Res 2004;16:288-93.

26. Mulhall JP, Branch J, Lubrano T, Shankey TV. Perturbation of cell cycle regulators in Peyronie's disease. Int J Impot Res 2001;13(suppl 5):S21-8.

27. Mulhall JP, Thom J, Lubrano T, Shankey TV. Basic fibroblast growth factor expression in Peyronie's disease. J Urol 2001;165:419-23

28. Wynn TA. Cellular and molecular mechanisms of fibrosis.J Pathol 2008;214:199-210.

29. Hinz B, Phan SH, Thannickal VJ, Galli A, Bochaton-Piallat ML, Gabbiani G. The myofibroblast: One function, multiple origins. Am J Pathol 2007;170:1807-16.

30. Ehrlich HP. Scar contracture: Cellular and connective tissue aspects in Peyronie's disease. J Urol 1997;157:316-9.

31. Nolazco G, Kovanecz I, Vernet D, Gelfand RA, Tsao J, Ferrini MG, Magee T, Rajfer J, GonzalezCadavid NF. Effect of muscle-derived stem cells on the restoration of corpora cavernosa smooth muscle and erectile function in the aged rat. BJU Int 2008;101:1156-64.

32. Gonzalez-Cadavid NF. Mechanisms of penile fibrosis. J Sex Med 2009;6(suppl 3):353-62.

33. El-Sakka Al, Hassoba HM, Pillarisetty RJ, Dahiya $R$, Lue TF. Peyronie's disease is associated with an increase in transforming growth factor-beta protein expression. J Urol 1997;158:1391-4.

34. Lin CS, Lin G,Wang Z, Maddah SA, Lue TF. Upregulation of monocyte chemoattractant protein 1 and effects of transforming growth factor-beta 1 in Peyronie's disease. Biochem Biophys Res Commun 2002;295:1014-9.

35. Gur S, Limin M, Hellstrom WJ. Current status and new developments inPeyronie's disease: medical, minimally invasive and surgical treatmentoptions. Expert Opin Pharmacother 2011; 12: 931-44

36. Kadioglu A, Kucukdurmaz F, Sanli O. Current status of the surgicalmanagement of Peyronie's disease. Nat Rev Urol 2011; 8: 95-106.

37. Mulhall, J. P., Schiff, J. \& Guhring, P. An analysis of the natural history of Peyronie's disease. J. Urol. 175, 2115-2118 (2006).
38. Hellstrom, w. J. \& Bivalacqua, T. J. Peyronie's disease: etiology, medical and surgical therapy.J. Androl. 21, 347-354 (2000).

39. Levine LA, Greenfield JM. Establishing a standardized evaluation of the man with Peyronie's disease. Int J Impot Res 2003;15(suppl 5): S103-12.

40. Pryor JP, Ralph DJ. Clinical presentations of Peyronie's disease. Int J Impot Res 2002;14: 414-7.

41. Schwarzer U, Sommer F, Klotz T, Braun M, Reifenrath B, Engelmann U. The prevalence of Peyronie's disease: Results of a large survey. BJU Int 2001;88:727-30.

42. Levine, L. A. \& Greenfield, J. M. establishing a standardized evaluation of the man with Peyronie's disease. Int. J. Impot. Res. 15 (Suppl. 5), S103S112 (2003).

43. Smith, J. F., walsh, T. J. \& Lue, T. F. Peyronie's disease: a critical appraisal of current diagnosis and treatment. Int. J. Impot. Res. 20, 445-459 (2008).

44. Ohebshalom M, Mulhall J, Guhring P, Parker M. Measurement of penile curvature in Peyronie's disease patients: comparison of three methods. J Sex Med 2007; 4: 199.

45. Usta MF et al. (2004) Relationship between the severity of penile curvature and the presence of comorbidities in men with Peyronie's disease. $J$ Urol 171: 775-779.

46. Carrieri MP et al. (1988) A case-control study on risk factors for Peyronie's disease. J Clin Epidemio/51: 511-515.

47. Aversa A, Sarteschi LM. The role of penile colorduplex ultrasound for the evaluation of erectile dysfunction. J Sex Med 2007;4:1437-47.

48. Gholami SS, Gonzalez-Cadavid NF, Lin CS, Rajfer J, Lue TF. Peyronie's disease: a review. J Urol 2003; 169 1234-41.

49. Shindel AW, Bullock TL, Brandes S. Urologist practice patterns in the management of Peyronie's disease: A nationwide survey. J Sex Med 2008;5:954-64; Epub 2007 Nov 27.

50. Azzi A. Molecular mechanism of alpha-tocopherol action. Free Radic Biol Med 2007; 43: 16. 
51. Ralph DJ, Brooks MD, Bottazzo GF, Pryor JP. The treatment of Peyronie's disease with tamoxifen. Br J Urol 1992; 70:648-51.

52. Teloken C, Rhoden EL, Grazziotin TM, Ros CT, Sogari PR, Souto CA. Tamoxifen versus placebo in the treatment of Peyronie's disease. J Urol 1999;162:2003-5.

53. Akkus E, Carrier S, Rehman J, Breza J, Kadioglu A, Lue TF. Is colchicine effective in Peyronie's disease? A pilot study. Urology 1994;44:291-5.

54. Kadioglu A, Tefekli A, Köksal T, Usta M, Erol H. Treatment of Peyronie's disease with oral colchicine: Long-term results and predictive parameters of successful outcome. Int J Impot Res 2000;12:169-75.

55. Safarinejad MR. Therapeutic effects of colchicine in the management of Peyronie's disease: A randomized doubleblind, placebo-controlled study. Int J Impot Res 2004;16:238-43.

56. Takami M, Cho ES, Lee SY, Kamijo R, Yim M. Phosphodiesterase inhibitors stimulate osteoclast formation via TRANCE/ RANKL expression in osteoblasts: possible involvement of ERK and p38 MAPK pathways. FEBS Lett 2005; 579: 832.

57. Brant WO, Dean RC, Lue TF. Treatment of Peyronie's disease with oral pentoxifylline. Nat Clin Pract Urol 2006; 3:111-5, 37.

58. Wagenknecht LV. Differential therapies in various stages of penile induration. Arch Esp Urol 1996; 49: 285.

59. Carson CC. Potassium para-aminobenzoate for the treatment of Peyronie's disease: is it effective? Tech Urol 1997; 3: 135.

60. Bremer J. Carnitine-metabolism and functions. Physiol Rev 1983; 63: 1420.

61. Biagiotti G, Cavallini G. Acetyl-L-carnitine vs tamoxifen in the oral therapy of Peyronie's disease: a preliminary report. BJU Int 2001; 88: 63.

62. Winter CC, Khanna R. Peyronie's disease: Results with dermo-jet injection of dexamethasone. J Urol 1975;114:898-900.

63. Jordan $\mathrm{GH}$. The use of intralesional clostridial collagenase injection therapy for Peyronie's disease: A prospective, single-center, nonplacebocontrolled study. J Sex Med 2008; 5: 180-7.
64. Levine LA, Merrick PF, Lee RC. Intralesional verapamil injection for the treatment of Peyronie's disease. J Urol 1994;151:1522-24.

65. Hellstrom WJ, Kendirci M, Matern R, Cockerham Y, Myers L, Sikka SC, Venable D, Honig S,McCullough A, Hakim LS, NehraA. Single-blind, multicenter, placebo controlled, parallel study to assess the safety and efficacy of intralesional interferon alpha-2B for minimally invasive treatment for Peyronie's disease. J Urol 2006;176:394-8.

66. Montorsi F, Salonia A, Maga T et al. Evidence based assessment of long-term results of plaque incision and vein grafting for Peyronie's disease. $J$ Urol 2000; 163: 1704-8.

67. David Ralph, MD, Nestor Gonzalez-Cadavid, PhD, Vincenzo Mirone, MD, Sava Perovic, MD, Michael Sohn, MD, Mustafa Usta, MD and Laurence Levine, MD, The Management of Peyronie's Disease: Evidence-based 2010 Guidelines, Ralph et al. J Sex Med 2010;7:2359-2374.

68. Nesbit RM. Congenital curvature of the phallus: report of three cases with description of corrective operation. J Urol 1965; 93: 230-2.

69. Pryor JP, Fitzpatrick JM. A new approach to the correction of the penile deformity in Peyronie's disease. J Urol 1979; 122: 622-3.

70. Savoca G, Thrombetta C, Ciampalini S, De Stefani $\mathrm{S}$, Buttazzi L, Belgrano E. Long-term results with Nesbit's procedure as treatment of Peyronie's disease. Int J Impotence Res 2000; 12: 289-93.

71. Syed AH, Abbasi Z, Hargreave TB. Nesbit procedure for disabling Peyronie's curvature. a median followup of 84 months. Urology 2003; 61: 999-1003.

72. Licht MR, Lewis RW. Modified Nesbit procedure for the treatment of Peyronie's disease: a comparative outcome analysis.J Urol 1997; 158: 460-3.

73. Yachia D. Modified corporoplasty for the treatment of penile curvature. J Urol 1990; 143: 80-2.

74. Daitch JA, Angermeier KW, Montague DK. Modified corporoplasty for penile curvature. long term results and patient satisfaction. J Urol 1999; 162: 2006-9.

75. Kovac JR, Brock GB. Surgical outcomes and patient satisfaction after dermal, pericardial, and small intestinal submucosal grafting for Peyronie's disease. J Sex Med 2007;4:1500-8. 
76. Backhaus BO, Muller SC, Albers P. Corporoplasty for advanced Peyronie's disease using venous and/ or dermis grafting. new surgical technique and longterm patient satisfaction. J Urol 2003; 169: 981-4

77. Yurkanin JP, Dean R, Wessells H. Effect of incision and saphenous vein grafting for Peyronie's disease on penile length and sexual satisfaction. $\mathrm{J}$ Urol 2001; 166: 1769-72.

78. Hellstrom WJ, Usta MF. Surgical approaches for advanced Peyronie's disease patients. Int J Impot Res 2003; 15(Suppl 5): S121.

79. Mulcahy JJ, Wilson SK. Management of Peyronie's disease with penile prostheses. Int J Impot Res 2002; 14: 384.
80. Montorsi F, Guazzoni G, Bergamaschi F, Rigatti P. Patient-partner satisfaction with semi-rigid penile prosthesis for Peyronie's disease: a 5-year followup study. J Urol 1993; 150: 1819-21.

81. Carson CC. Penile prosthesis implantation in the treatment of Peyronie's disease and erectile dysfunction. Int J Impot Res 2000; 12(Suppl 4): S122.

Abbreviations:

CIS : Cavernosal Injection and self stimulation

ED : Erectile dysfunction

PD : Peyronie's Disease 